Portland State University

PDXScholar

8-1-1996

\title{
Independent center, independent electron approximation for dynamics of molecules and clusters
}

James H. McGuire

Jack C. Straton

Portland State University, straton@pdx.edu

J. Wang

Y. D. Wang

O. L. Weaver

See next page for additional authors

Follow this and additional works at: https://pdxscholar.library.pdx.edu/phy_fac

Part of the Physics Commons

Let us know how access to this document benefits you.

\section{Citation Details}

Independent center, independent electron approximation for dynamics of molecules and clusters, J. $\mathrm{H}$. McGuire, Jack C. Straton, and J. Wang, Y. D. Wang and O. L. Weaver, S. E. Corchs and R. D. Rivarola, J. Chemical Physics 105, 1846 (1996). http://dx.doi.org/10.1063/1.472816

This Article is brought to you for free and open access. It has been accepted for inclusion in Physics Faculty Publications and Presentations by an authorized administrator of PDXScholar. Please contact us if we can make this document more accessible: pdxscholar@pdx.edu. 
Authors

James H. McGuire, Jack C. Straton, J. Wang, Y. D. Wang, O. L. Weaver, S. E. Corchs, and R. D. Rivarola 


\title{
Independent center, independent electron approximation for dynamics of molecules and clusters
}

\author{
J. H. McGuire, Jack C. Straton, and J. Wang \\ Department of Physics, Tulane University, New Orleans, Louisianna 70118-5698
}

Y. D. Wang and O. L. Weaver

Department of Physics, Kansas State University, Manhattan, Kansas 66506

\author{
S. E. Corchs and R. D. Rivarola \\ Instituto de Fisica Rosario, CONICET - Universidad Nacional de Rosario, 2000 Rosario, Argentina
}

(Received 14 February 1996; accepted 16 April 1996)

\begin{abstract}
A formalism is developed for evaluating probabilities and cross sections for multiple-electron transitions in scattering of molecules and clusters by charged collision partners. First, the molecule is divided into subclusters each made up of identical centers (atoms). Within each subcluster coherent scattering from identical centers may lead to observable phase terms and a geometrical structure factor. Then, using a mean field approximation to describe the interactions between centers we obtain $A_{I} \sim \sum_{k} \Pi_{k} e^{i \delta_{I}^{k}} A_{I k}$. Second, the independent electron approximation for each center may be obtained by neglecting the correlation between electrons in each center. The probability amplitude for each center is then a product of single electron transition probability amplitudes, $a_{I k}^{i}$, i.e. $A_{I k} \approx \prod_{i} a_{i k}^{i}$. Finally, the independent subcluster approximation is introduced by neglecting the interactions between different subclusters in the molecule or cluster. The total probability amplitude then reduces to a simple product of amplitudes for each subcluster, $A \approx \Pi_{I} A_{I}$. Limitations of this simple approximation are discussed. (c) 1996 American Institute of Physics.
\end{abstract} [S0021-9606(96)00728-3]

\section{INTRODUCTION}

Understanding interactions of few and many electron systems is central to detailed understanding of physical and chemical properties of microscopic and macroscopic atomic and molecular systems. Even on the scale of individual atoms, detailing the nature of both static and dynamic observables is limited by the difficulty of evaluating few and many electron effects. In general, the larger the system the greater the difficulty. While, in principle, properties of micro- and macro-structures depend on atomic properties, in practice understanding large atomic and molecular systems is limited by the lack of methods that are simple enough to be used for large systems of atoms and molecules. The purpose of this paper is to define a dynamic independent particle model for interactions of molecules and clusters with charged collision partners. Our model describes multiple electron transitions. We also address when and how well such a simple independent particle model works.

The simple independent electron approximation is now widely used to describe atomic collisions. ${ }^{1,2}$ Some reactions of simple molecules with fast ions have also been described. ${ }^{2-4}$ In this paper we introduce an independent particle model for molecules interacting with charged particles, so that one may, under certain conditions, evaluate probabilities, cross sections and reaction rates for systems of molecules in which more than one electron is active. Our method yields a probability for multi-electron transition that is expressed as a simple product of independent single center, single electron probabilities.

Classically the probability, $P_{12}$, that two independent events occur is the simple product of the individual probability, $P_{1}$ and $P_{2}$, for each event, i.e. $P_{12}=P_{1} \cdot P_{2}$. This simple idea was used to describe the dynamics of individual atoms interacting with heavy ions 25 years ago ${ }^{5,6}$ and a quantum derivation of such a result was first given in 1977. ${ }^{7}$ A key approximation required for such an independent electron approximation is to neglect the electron-electron correlation which interconnects the independent electron probabilities, $P_{1}$ and $P_{2}$. This independent electron approximation for atomic scattering has been generalized to systems with arbitrary numbers of electrons undergoing transitions, and has been widely tested experimentally. ${ }^{2}$ It is usually valid for atomic collisions in which electron correlation is weak and the interaction is sufficiently fast that complex correlated processes are unlikely.

For transitions of a single electron treating interactions of molecules with charged particles in terms of independent atomic electrons was discussed sometime ago by Landau and Liftshitz, ${ }^{4}$ by Zare, ${ }^{8}$ and also by Tuan and Gerjuoy. ${ }^{9}$ However, understanding and analysis of interactions of molecules and clusters often involves transitions of more than one electron. A more specific example of a case in which multiple electron transitions may be significant is Coulomb explosions $^{10-15}$ in which a molecule or cluster is quickly stripped of some of its electrons and breaks into mutually repulsive fragments. So processes involving multielectron transitions are clearly of interest. Nevertheless, until now most theoretical descriptions of such molecular dynamics have been limited to systems in which there is a single active electron. ${ }^{3}$ In this paper we introduce a method to describe collisions in which many electrons may undergo transitions. 
The basic idea of this paper is to set forth a method to evaluate multiple electron transition probabilities and cross sections in large molecules and clusters interacting with charged particles using an independent electron approximation where whatever happens to one electron does not influence the other electrons. The electronic wave function for an atom or center is written as a simple product of single orthogonal electron wave functions, each of which evolves independently. Atoms or centers with the same wave functions may be grouped into subclusters. In these subclusters the interactions between these atoms or centers are neglected. However, the transition amplitude is taken to be a sum of transition amplitudes for each atom or center with a translational phase. Finally the subcluster wave functions are multiplied together independently to form the electronic wave function for the final molecule or cluster. While most molecules are not of this form, the methods we develop may be applied to many molecules using sensible combinations of one or all of the three primary approximations we develop in this paper. The purpose of this paper is to determine under what conditions such simple approximations are valid. Mathematically and physically it is sensible to begin with the exact Hamiltonian for the full molecule or cluster and break it down to the level of independent electrons in successive approximations. That is how we proceed.

The limitations of our independent particle approach depend on the validity of the approximations we employ, namely largely neglecting electron correlation and exchange. While use of these approximations simplifies the many body problem both mathematically and conceptually, effects such as chemical bonding that depend on correlation and exchange, which are often important in molecular dynamics, will not be fully accounted for in our methods. On the other hand, our approach may provide a conceptually simple and analytically convenient method to understand the dynamics of multi-atom, multi-electron systems.

\section{THEORY}

Consider a molecule or cluster denoted by $C_{1}^{N_{1}} C_{2}^{N_{2}} \ldots C_{I}^{N_{I}} \ldots C_{N}^{N_{N}}$ where $C_{I}^{N_{I}}$ is one of $N$ different subclusters. The subcluster of kind $I$ is composed by $N_{I}$ identical centers $C_{I}^{k}\left(1 \leqslant k \leqslant N_{I}\right)$. Each center has one or more electrons. It is the activity of one or more of these electrons in which we are interested. In our model both the static and the dynamic properties of these electrons will be defined within each center independently. Each center, $C_{I}^{k}$, of the subcluster $I$ has the same nuclear charge $Z_{I}$ and nuclear mass $M_{I}$, and its center of mass is located a distance $\mathbf{R}_{I}^{k}$ from the center of mass of the molecule. Each individual center, $C_{I}$, carries $n_{I}$ electrons. This molecule interacts with a projectile of charge $Z_{P}$ and mass $M$, moving at a velocity $\mathbf{v}$, as illustrated in Fig. 1.

For clarity, we use the following development. We begin with the Hamiltonian describing a particular subcluster of identical centers (atoms). The formally exact transition amplitude is derived. Then, successive stages of approximation aimed at reducing the transition amplitude to a manageable

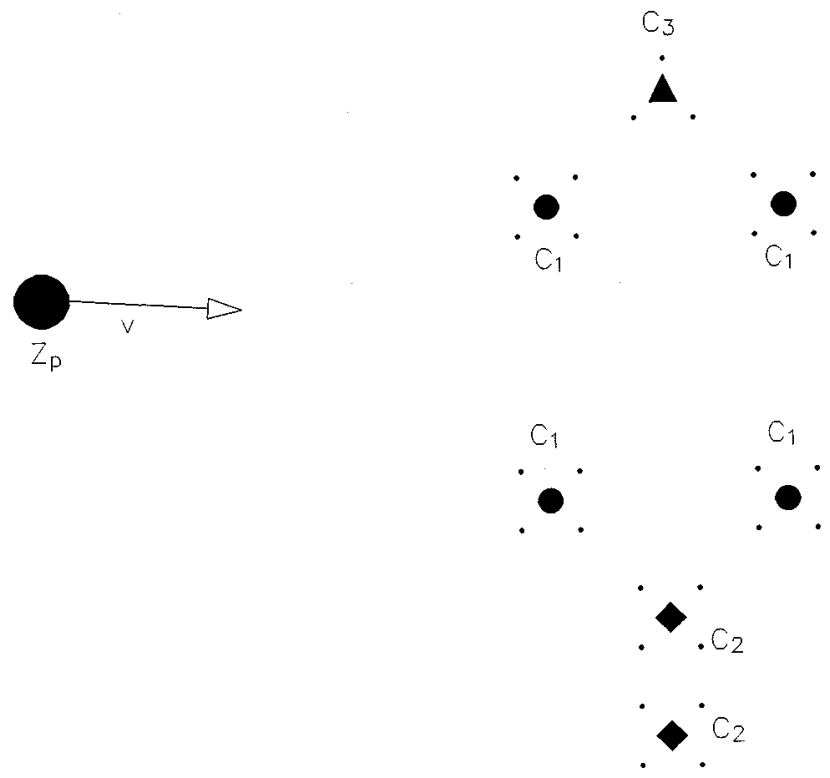

FIG. 1. A cluster of atomic centers in a collision with a projectile of charge, $Z_{p}$, and velocity, $v$. In the target cluster there are four identical subcluster $C_{1}$ centers, two subcluster $C_{2}$ centers and one subcluster $C_{3}$ center.

form are applied as follows. First, independent center approximation is applied to the subcluster in which correlation among the centers is averaged out. The resulting transition amplitude is given as a product of amplitudes for each of the centers. Optional geometrical factors are also considered. Second, independent electron approximation is used within each center, reducing the transition amplitude on each center to a product of amplitudes for single-electron transitions. Then, an example is given to illustrate the concepts developed in the first two approximations. Finally, generalization to the whole cluster is made by treating subclusters independent of each other, yielding the total transition amplitude as a product of individual subcluster amplitudes.

\section{A. Exact formulation}

The Hamiltonian of a subcluster $I$, using atomic units $\left(e^{2}=\hbar=m_{e}=1\right)$ and working in the laboratory system, is

$$
H=K+V+H_{0, I} .
$$

Here

$$
K=-\frac{\nabla^{2}}{2 M}
$$

is the kinetic energy of the projectile in the center of mass of the molecule, $\mathrm{V}$ the interaction of the projectile with the subcluster given by

$$
V=Z_{P} \sum_{k=1}^{N_{I}}\left\{\frac{Z_{I}}{\left|\mathbf{R}-\mathbf{R}_{I}^{k}\right|}-\sum_{i=1}^{n_{I}} \frac{1}{\left|\mathbf{R}-\mathbf{R}_{I}^{k}-\mathbf{r}_{I}^{k, i}\right|}\right\},
$$




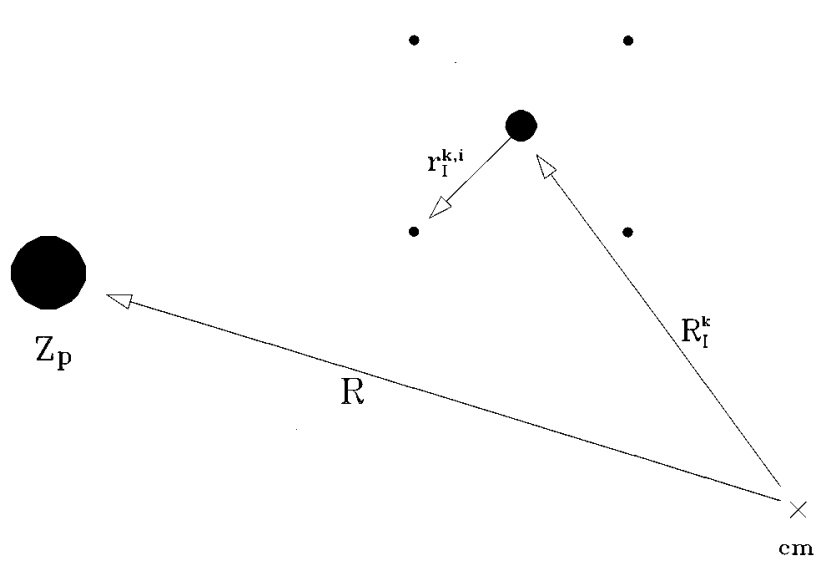

FIG. 2. Definition of coordinates used in the text. The center-of-mass of the cluster is denoted "cm". The center shown above corresponds to the upper left $C_{1}$ center in Fig. 1. The index $\mathrm{K}$ (not shown) runs over different subclusters (e.g. $C_{1}$ and $C_{2}$ in Fig. 1). The index k runs over members of the same subcluster (e.g. $\mathrm{K}$ runs from 1 to 4 in the subcluster containing 4 $C_{1}$ 's in Fig. 1). The index i runs over the electrons in a given center (e.g. in the above figure the index i runs from 1 to 4 ).

where $\mathbf{R}$ is the position of the projectile with respect to the center of mass of the molecule and $\mathbf{r}_{I}^{k, i}$ is the coordinate of the $i$ th electron of the center $C_{I}^{k}$ with respect to its nucleus, as illustrated in Fig. 2.

Also, in Eq. (1) $H_{0, I}$ is the Hamiltonian of the static subcluster of type I given by,

$$
\begin{aligned}
H_{0, I}= & \sum_{k=1}^{N_{I}}\left\{-\frac{\nabla_{\mathbf{R}_{I}^{k}}^{2}}{2 M_{I}}+\sum_{i=1}^{n_{I}}\left[-\frac{\nabla_{\mathbf{r}_{I}^{k, i}}^{2}}{2}-\frac{Z_{I}}{r_{I}^{k, i}}\right.\right. \\
& \left.\left.+\frac{1}{2} \sum_{\substack{l=1 \\
(l \neq i)}}^{n_{I}} \frac{1}{\left|\mathbf{r}_{I}^{k, i}-\mathbf{r}_{I}^{k, I}\right|}\right]\right\} \\
& +\sum_{k=1}^{N_{I}} \sum_{\substack{j=1 \\
(j \neq k)}}^{N_{I}}\left\{\frac{1}{2} \frac{Z_{I}^{2}}{\left|\mathbf{R}_{I}^{k}-\mathbf{R}_{I}^{j}\right|}+\sum_{i=1}^{n_{I}}\left[-\frac{Z_{I}}{\left|\mathbf{R}_{I}^{k}-\mathbf{R}_{I}^{j}-\mathbf{r}_{I}^{j, i}\right|}\right.\right. \\
& \left.\left.+\frac{1}{2} \sum_{l=1}^{n_{I}} \frac{1}{\left|\mathbf{R}_{I}^{k}+\mathbf{r}_{I}^{k, i}-\mathbf{R}_{I}^{j}-\mathbf{r}_{I}^{j, l}\right|}\right]\right\}
\end{aligned}
$$

In order to develop a model with independent centers, we regard the first line in Eq. (4) above as the sum of the Hamiltonians of each individual center that belongs to the subcluster I. Line two contains the sum of the interactions between these atomic centers: (i) nucleus-nucleus interaction between $C_{I}^{k}$ and $C_{I}^{j}$, (ii) the interaction term between the nucleus of $C_{I}^{k}$ and the electrons of $C_{I}^{j}$, and (iii) electronelectron interaction (or correlation) between electrons of $C_{I}^{k}$ and those of $C_{I}^{j}$.

The Schrödinger equation to solve is given by

$$
(H-E) \Psi_{i, f}^{+,-}=0,
$$

where $E$ is the total energy of the system and $\Psi_{i}^{+}\left(\Psi_{f}^{-}\right)$is the exact solution of Eq. (7) with correct outgoing (incoming) conditions corresponding to the entry (exit) channel.

In order to separate the projectile motion from the electronic motion we introduce eikonal phases to describe the scattering between the projectile nucleus and each of the nuclei of the subcluster. ${ }^{16}$ In the entry channel we write

$$
\begin{aligned}
\Psi_{i}^{+} & =\left[\prod_{k=1}^{N_{I}} \exp \left\{i \frac{Z_{P} Z_{I}}{v} \ln \left[k_{i}\left|\mathbf{R}-\mathbf{R}_{I}^{k}\right|-\mathbf{k}_{i} \cdot\left(\mathbf{R}-\mathbf{R}_{I}^{k}\right)\right]\right\}\right] \\
& \times \exp \left\{i \mathbf{k}_{i} \cdot \mathbf{R}\right\} \exp \left\{i \frac{\epsilon_{i} Z}{v}\right\} \psi_{i}^{+}
\end{aligned}
$$

In the same way, for the exit channel we write

$$
\begin{aligned}
\Psi_{f}^{-}= & {\left[\prod _ { k = 1 } ^ { N _ { I } } \operatorname { e x p } \left\{-i \frac{Z_{P} Z_{I}}{v_{f}} \ln \left[k_{f}\left|\mathbf{R}-\mathbf{R}_{I}^{k}\right|\right.\right.\right.} \\
& \left.\left.\left.-\mathbf{k}_{f} \cdot\left(\mathbf{R}-\mathbf{R}_{I}^{k}\right)\right]\right\}\right] \exp \left\{i \mathbf{k}_{f} \cdot \mathbf{R}\right\} \exp \left\{i \frac{\epsilon_{f} Z}{v_{f}}\right\} \psi_{f}^{-} .
\end{aligned}
$$

In Eqs. (6) and (7) $\mathbf{k}_{i}=M \mathbf{v}$ and $\mathbf{k}_{f}=M_{f} \mathbf{v}_{f}$ denote the initial and final momenta of the projectile respectively. Also, $Z$ is the component of $\mathbf{R}$ in the direction of the vector $\mathbf{v}$ and we have introduced the energies $\epsilon_{i}$ and $\epsilon_{f}$ that satisfy

$$
E=\frac{k_{i}^{2}}{2 M}+\epsilon_{i}=\frac{k_{f}^{2}}{2 M_{f}}+\epsilon_{f} .
$$

Working within the eikonal approximation, we apply the operator $(H-E)$ on $\Psi_{i, f}^{+,-}$given by (6) and (7) and the Schrödinger equation for the electronic motion is obtained

$$
\left(H_{\mathrm{el}}-i \frac{\partial}{\partial t}\right) \psi_{i, f}^{+,-}=0
$$

where the Hamiltonian governing the evolution of the electrons in the subcluster, $H_{\mathrm{el}}$, is defined as

$$
H_{e l}=H_{0, I}+V-\sum_{k=1}^{N_{I}} \frac{Z_{P} Z_{I}}{\left|\mathbf{R}-\mathbf{R}_{I}^{k}\right|}=H_{0, I}+V^{\prime}
$$

and $\psi_{i, f}^{+,-}$is the time dependent wave function with correct outgoing and incoming conditions that describes the electronic motion. The Born-Oppenheimer approximation is used and we regard the $\mathbf{R}_{I}^{k}$ as fixed. Next it is assumed that the projectile motion may be treated classically ${ }^{17}$ so that the projectile trajectory $\mathbf{R}(t)$ is well defined. The simplest (but not the only possible) trajectory is $\mathbf{R}(t)=\mathbf{b}+\mathbf{v} t$, where $\mathbf{b}$ is the impact parameter of the projectile relative to the center of mass of the molecule.

In Eq. (10) the potential $V^{\prime}$ is the sum of the interactions of the projectile with each of the target electrons given by

$$
V^{\prime}=\sum_{k=1}^{N_{I}} \sum_{i=1}^{n_{I}} V_{I}^{k, i}=\sum_{k=1}^{N_{I}} \sum_{i=1}^{n_{I}} \frac{-Z_{P}}{\left|\mathbf{R}(t)-\mathbf{R}_{I}^{k}-\mathbf{r}_{I}^{k, i}\right|}
$$

If we define 


$$
V_{I}^{k}=\sum_{i=1}^{n_{I}} V_{I}^{k, i}
$$

and

$$
V_{I}=\sum_{k=1}^{N_{I}} V_{I}^{k}
$$

then

$$
V^{\prime} \equiv V_{I}
$$

It is now advantageous to work in the intermediate representation where one may take advantage of the fact that the eigenfunctions of $H_{0, I}$ are known (or nearly known). In the intermediate representation the evolution operator $U\left(t, t_{0}\right)$ is governed by

$$
i \frac{d U\left(t, t_{0}\right)}{d t}=V^{\prime}(t) U\left(t, t_{0}\right)
$$

where

$$
V^{\prime}(t)=e^{i H_{0, I^{t}}} V^{\prime} e^{-i H_{0, I^{t}}} .
$$

Here $V^{\prime}(t)$ is not a sum of single electron (or single center) operators because $H_{0, I}$ in Eq. (4) is not a sum of single electron (or single center) terms due to the correlation interactions between the electrons (or centers). Equation (9) may be formally solved using the time ordering operator, $T$, namely

$$
U\left(t, t_{0}\right)=T \exp \left[-i \int_{t_{0}}^{t} V^{\prime}(t) d t\right] .
$$

The probability amplitude for transition of electrons in the asymptotic initial state $\phi_{i}$ to the asymptotic final state $\phi_{f}$ of the molecule or cluster is found by projecting the full electronic wave function of Eq. (9) satisfying initial boundary conditions, $\psi_{i}^{+}$, onto the asymptotic electronic wave function $\phi_{f}$, namely, 2,18

$$
A=\left\langle\phi_{f} \mid \psi_{i}^{+}\right\rangle=\left\langle\phi_{f}|U(+\infty,-\infty)| \phi_{i}\right\rangle .
$$

The probability $P(\mathbf{b})$ for a transition from $\phi_{i}$ to $\phi_{f}$ is given by the absolute square of $A$, and the corresponding cross section is found by a two dimensional integration over the impact parameter, b, namely,

$$
\sigma=\int P(\mathbf{b}) d \mathbf{b}=\int|A|^{2} d \mathbf{b} .
$$

This result holds for an arbitrary number of centers and an arbitrary number of electrons. It is formally exact. Successive approximations to Eqs. (17) and (18) are developed in the the next subsections.

\section{B. The independent center approximation}

In this subsection we decouple the centers within each subcluster. Also we include the optional possibility of expressing the probability amplitude, $A_{I}$, for each subcluster as a sum of products of the probability amplitudes, $A_{I}^{k}$, for electronic transitions on each constituent center, $C_{I}^{k}$. In this sum phases due to the translation between the centers are retained.
In order to obtain the independent center approximation we introduce an average potential, $\mathscr{T}\left(\mathbf{r}_{I}^{k, i}\right)$, so that the Hamiltonian $H_{0, I}$ given by Eq. (4) is approximated by

$$
\begin{aligned}
H_{0, I} \cong & \sum_{k=1}^{N_{I}}\left\{-\frac{\nabla_{\mathbf{R}_{I}^{k}}^{2}}{2 M_{I}}+\sum_{i=1}^{n_{I}}\left[-\frac{\nabla_{\mathbf{r}_{I}^{k, i}}^{2}}{2}+\mathscr{V}\left(\mathbf{r}_{I}^{k, i}\right)\right.\right. \\
& \left.\left.+\frac{1}{2} \sum_{\substack{l=1 \\
(l \neq i)}}^{n_{I}} \frac{1}{\left|\mathbf{r}_{I}^{k, i}-\mathbf{r}_{I}^{k, l}\right|}\right]\right\} \equiv \sum_{k=1}^{N_{I}} h_{I, k},
\end{aligned}
$$

where the term $\Sigma_{i} \mathscr{T}\left(\mathbf{r}_{I}^{k, i}\right)$ results from the following approximation

$$
\begin{aligned}
\sum_{i=1}^{n_{I}} \mathscr{V}\left(\mathbf{r}_{I}^{k, i}\right) \cong & \sum_{i=1}^{n_{I}}\left\{-\frac{Z_{I}}{r_{I}^{k, i}}+\left\langle\sum _ { \substack { j = 1 \\
( j \neq k ) } } ^ { N _ { I } } \left[-\frac{Z_{I}}{\left|\mathbf{R}_{I}^{j}-\mathbf{R}_{I}^{k}-\mathbf{r}_{I}^{k, i}\right|}\right.\right.\right. \\
& \left.\left.\left.+\frac{1}{2} \sum_{l=1}^{n_{I}} \frac{1}{\left|\mathbf{R}_{I}^{j}+\mathbf{r}_{I}^{j, i}-\mathbf{R}_{I}^{k}-\mathbf{r}_{I}^{k, l}\right|}\right]\right\rangle\right\}
\end{aligned}
$$

where \langle\rangle denotes averaging of the interactions between centers by mean field approximation. Let us note that it is not necessary to include the nucleus-nucleus interaction between $C_{I}^{k}$ and $C_{I}^{j}$ in $\Sigma_{i} \mathscr{V}\left(\mathbf{r}_{I}^{k, i}\right)$ if the nuclei of the centers are regarded as frozen during the collision.

Then, the $h_{I, k}$ terms defined in Eq. (20) are indeed single center operators satisfying $\left[h_{I, k}, H_{0, I}\right]=0$. Recalling that $V_{I}=\Sigma V_{I}^{k}$ from Eq. (13) we have using Eq. (16) that

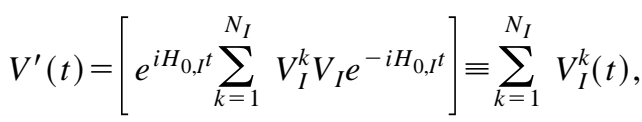

where $V_{I}^{k}(t)$ now operates on a single center.

Using Eq. (17) for the evolution operator, one now has,

$$
\begin{aligned}
U\left(t, t_{0}\right) \equiv U_{I}\left(t, t_{0}\right) & =T \exp \left[-i \sum_{k=1}^{N_{I}} \int_{t_{0}}^{t} V_{I}^{k}(t) d t\right] \\
& =\prod_{k=1}^{N_{I}} U_{I}^{k}\left(t, t_{0}\right)
\end{aligned}
$$

where

$$
U_{I}^{k}\left(t, t_{0}\right)=T \exp \left[-i \int_{t_{0}}^{t} V_{I}^{k}(t) d t\right] .
$$

Now the centers evolve independently. Then, the initial wave function of the subcluster $I, \phi_{I, i}$, can be written as a product of wave functions $\varphi_{k i}\left(\mathbf{R}_{I}^{k}\right)$ of $h_{I k}$ (the subindex $k i$ indicates the initial state of center $\left.C_{I}^{k}\right)$

$$
\phi_{I i}=\prod_{k=1}^{N_{I}} \varphi_{k i}\left(\mathbf{R}_{I}^{k}\right) \text {. }
$$

Assuming that the final state of each one of the centers of the subcluster $I$ is known, the final wave function $\phi_{I f}$ reads

$$
\phi_{I f}=\prod_{k=1}^{N_{I}} \varphi_{k f}\left(\mathbf{R}_{I}^{k}\right),
$$


where $k f$ indicates the final state of center $C_{I}^{k}$. From Eqs. (18), (23), (25), and (26) we have that

$$
\begin{aligned}
A_{I} & =\left\langle\prod_{k=1}^{N_{I}} \varphi_{k f}\left(\mathbf{R}_{I}^{k}\right)\left|\prod_{k=1}^{N_{I}} U_{I}^{k}\left(t, t_{0}\right)\right| \prod_{k=1}^{N_{I}} \varphi_{k i}\left(\mathbf{R}_{I}^{k}\right)\right\rangle \\
& \equiv \prod_{k=1}^{N_{I}} A_{I k}\left(\mathbf{R}_{I}^{k}\right),
\end{aligned}
$$

where

$$
A_{I k}\left(\mathbf{R}_{I}^{k}\right)=\left\langle\varphi_{k f}\left(\mathbf{R}_{I}^{k}\right)\left|U_{I}^{k}\left(t, t_{0}\right)\right| \varphi_{k i}\left(\mathbf{R}_{I}^{k}\right)\right\rangle .
$$

At this point we have simply expressed the subcluster probability amplitude as a simple product of probability amplitudes for each of the constituent centers.

Let us now assume that the transition from state $\varphi_{k i}$ to state $\varphi_{k f}$ can be distinguished while we ignore in which of the $N_{I}$ identical centers it has occurred. The probability amplitude $A_{I}$ in the subcluster $I$ is then evaluated as

$$
A_{I}=\frac{1}{N_{I} !} \sum_{P} P \prod_{k=1}^{N_{I}} A_{I k}\left(\mathbf{R}_{I}^{k}\right)
$$

where the operator $\Sigma_{P} P$ indicates the sum over all the possible permutations between the transitions $k i \rightarrow k f$ and the centers $C_{I}^{k}$ of the subcluster in which they occurred. For example, if $N_{I}=2$ then Eq. (29) reads

$$
A_{I}=\frac{1}{2}\left\{A_{I 1}\left(\mathbf{R}_{I}^{1}\right) A_{I 2}\left(\mathbf{R}_{I}^{2}\right)+A_{I 1}\left(\mathbf{R}_{I}^{2}\right) A_{I 2}\left(\mathbf{R}_{I}^{1}\right)\right\} .
$$

Let us now consider the single center probability amplitude, $A_{I k}\left(\mathbf{R}_{I}^{k}\right)$ corresponding to the transition $\varphi_{k i} \rightarrow \varphi_{k f}$. The amplitude $A_{I k}$, evaluated at $\mathbf{R}_{I}^{k}$ and as a function of the impact parameter $\mathbf{b}$, is related to another amplitude evaluated at some other point, $\mathbf{R}_{0}$, in space by a phase due to translation in time, ${ }^{2,19-21}$ namely

$$
A_{I k}\left(\mathbf{R}_{I}^{k}\right)=e^{-i \delta_{I}^{k}} A_{I k}\left(\mathbf{R}_{0}\right),
$$

where

$$
\delta_{I}^{k}=Q_{z}^{k}\left(R_{I z}^{k}-R_{0 z}\right),
$$

with $Q_{z}^{k}$ the $z$ component of $\mathbf{Q}$, the momentum transferred to the projectile in the transition $\varphi_{k i} \rightarrow \varphi_{k f}$. The $z$ axis is taken parallel to the velocity of the incoming projectile at large distances. Also, $\left(R_{I z}^{k}-R_{0 z}\right)$ is the $z$ component of $\left(\mathbf{R}_{I}^{k}-\mathbf{R}_{0}\right)$. Here we choose the $\mathbf{R}_{0}$ as the center of mass of the molecule and set $\mathbf{R}_{0}=0$. For a given center, $C_{I}^{k}$, the impact parameter in $A_{I k}(0)$ is the impact parameter of the projectile relative to the nucleus of $C_{I}^{k}$, i.e. $\mathbf{b}_{I}^{k}=\mathbf{b}-\mathbf{R}_{I \perp}^{k}$ where $\mathbf{R}_{I \perp}^{k}=\mathbf{R}_{I}^{k}-\mathbf{R}_{I z}^{k}$. For heavy projectiles with $M \gg m_{e}$, one has, $Q_{z}^{k} \cong Q_{\min }^{k}$ where $Q_{\min }^{k}$ is the minimum momentum transferred to the projectile. If an excitation process takes place in center $C_{I}^{k}$ we have that $Q_{\min }^{k}=\Delta E^{k} / 2 v$ where $\Delta E^{k}$ is the energy gain of the electrons in the transition $\varphi_{k i} \rightarrow \varphi_{k f}$ and in the electron capture case $Q_{\min }^{k}=v / 2-\Delta E^{k} / v$, as given by McDowell and Coleman. ${ }^{22}$

From Eqs. (29) and (31) we obtain

$$
A_{I}=\frac{1}{N_{I} !} \sum_{P} P \prod_{k} \exp \left\{-i Q_{\min }^{k} R_{I z}^{k}\right\} A_{I k}\left(\mathbf{b}_{I}^{k}\right) .
$$

Equation (33) is the main equation of this subsection. The phase terms lead to the geometrical structure factor as discussed in Sec. III. C.

\section{The independent electron approximation}

In this subsection we shall remove the interaction between electrons on each independent center and obtain the independent center independent electron approximation. ${ }^{2,3,7,18}$

As we did in subsection B we introduce an effective potential so that the single center Hamiltonian $h_{I, k}$ given by Eq. (20) is approximated by

$$
h_{I, k} \cong \sum_{l=1}^{n_{I}}\left[-\frac{\nabla_{\mathbf{r}_{I}^{k, l}}^{2}}{2}+V_{e f}\left(\mathbf{r}_{I}^{k, l}\right)\right] \equiv \sum_{l=1}^{n_{I}} h_{I, k}^{l},
$$

where the term $\Sigma_{l} V_{\mathrm{ef}}\left(\mathbf{r}_{I}^{k, l}\right)$ gives a mean field approximation to the non-local electron-electron interactions, namely,

$$
\sum_{l=1}^{n_{I}} V_{\mathrm{ef}}\left(\mathbf{r}_{I}^{k, l}\right) \cong \sum_{l=1}^{n_{I}}\left\{\mathscr{T}\left(\mathbf{r}_{I}^{k, l}\right)+\frac{1}{2}\left\langle\sum_{\substack{i=1 \\(i \neq l)}}^{n_{I}} \frac{1}{\left|\mathbf{r}_{I}^{k, l}-\mathbf{r}_{I}^{k, i}\right|}\right\rangle\right\} .
$$

In Eq. (34) the kinetic energy of the nucleus center has been neglected. This is valid in high velocity collisions for heavy projectiles where the collision is sufficiently fast so that the centers are effectively frozen in place during the collision.

From Eqs. (12) and (17), and using the fact that $h_{I, k}^{l}$ are single electron Hamiltonian terms, the evolution operator given by Eq. (24) reads

$$
U_{I}^{k}\left(t, t_{0}\right)=\prod_{l=1}^{n_{I}} U_{I}^{k, l}\left(t, t_{0}\right)
$$

where

$$
U_{I}^{k, l}\left(t, t_{0}\right)=T \exp \left[-i \int_{t_{0}}^{t} V_{I}^{k, l}(t) d t\right] .
$$

Now the Hamiltonian of center $C_{I}^{k}$ given by Eq. (34) is a sum of independent terms for each electron. Then, the electronic wave function for this center $\varphi_{k}$ is a product of wave functions $\phi_{k}^{l}$ for each electron, $l$. As a consequence, the probability amplitude $A_{I k}$ is a product of single electron probability amplitudes $a_{I k}^{l}$, namely

$$
A_{I k}=\prod_{l=1}^{n_{I}}\left\langle\phi_{k f}^{l}\left|U_{I}^{k, l}\left(t, t_{0}\right)\right| \phi_{k i}^{l}\right\rangle \equiv \prod_{l=1}^{n_{I}} a_{I k}^{l} .
$$

The effects of the exchange symmetry of electrons have not been taken into account in Eq. (38). In atomic collisions, these effects have been considered by Reading and Ford. ${ }^{23}$ At high collision velocities these exchange effects are often small and then may be neglected. It is often the case in large many electron systems that there are "passive" electrons which are not relevant to the processes under study. If these "passive" electrons are decoupled from the "active" elec- 


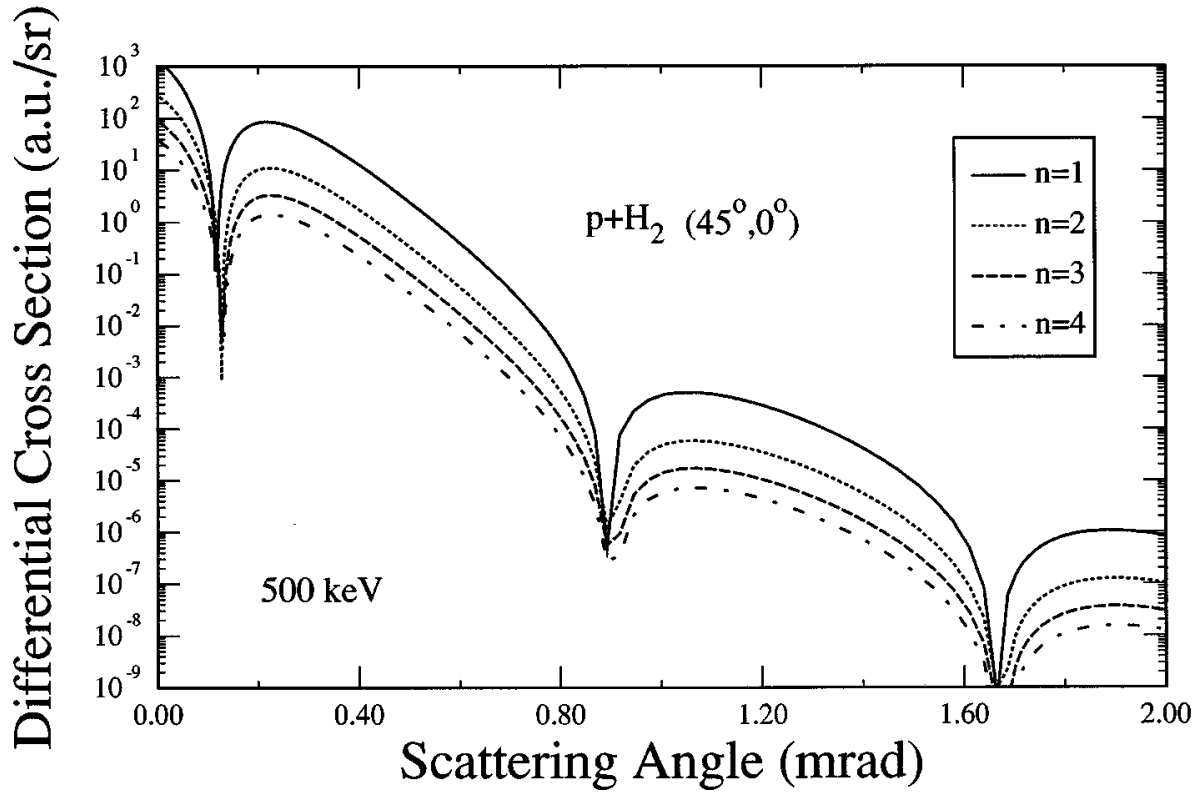

FIG. 3. Electron capture cross sections differential in projectile scattering angle by $500 \mathrm{keV}$ protons on $\mathrm{H}_{2}$. Capture into ground state $n=1$ and the first three excited states $n=2-4$ are included. The molecular orientation is space fixed at $45^{\circ}$ with respect to the beam direction in the collision plane $\left(0^{\circ}\right.$ azimuthal angle). The dips reflect interference patterns arising from scattering centers as described by the geometrical structure factor.

trons under consideration, then the total probability for all possible final states of the decoupled passive electrons sums to unity and their presence may be neglected. ${ }^{24}$

Finally, the independent center, independent electron approximation is obtained from Eqs. (29) and (38)

$$
A_{I} \cong \frac{1}{N_{I} !} \sum_{P} P \prod_{k=1}^{N_{I}} \exp \left\{-i Q_{\min }^{k} R_{I z}^{k}\right\} \prod_{l=1}^{n_{I}} a_{I k}^{l}\left(\mathbf{b}_{I}^{k}\right) .
$$

As a consequence of the approximations made in obtaining Eq. (39), this expression is only valid for electronic transitions from the inner shells of each one of the centers of the target (see discussion Sec. III).

\section{An illustrative example}

The two successive approximations derived from the preceding two sections for a given subcluster are applicable to a class of homonuclear molecules such as $\mathrm{H}_{2}, \mathrm{~N}_{2}, \mathrm{C}_{60}$, etc. In this subsection we give an example illustrating a few concepts developed so far.

We calculate electron capture from $\mathrm{H}_{2}$ by protons. Here the independent electron approximation becomes exact since there is only one electron on each center ( $\mathrm{H}$ atom). The cross section for a space-fixed orientation of the molecular axis may then be expressed ${ }^{3}$ as a product of the atomic cross section for capture from the $\mathrm{H}$ atom and the geometrical structure factor [see Eq. (39) and Sec. III C]. The capture cross section from atomic hydrogen is calculated by a first order method known as the Oppenheimer-BrinkmanKramers approximation. ${ }^{22}$ This approximation has been used $^{16}$ for studying electron capture as a function of the direction of the internuclear molecular axis, and agreement with experiments detecting Coulomb fragments was found. We note that it may also be calculated by other methods of any desired sophistication, since our formulation only asserts the concept of independent particle model and does not preclude in any way how the transition amplitudes can be obtained (they may even be taken from experimental data if so desired).

Shown in Fig. 3 is the cross section differential in projectile scattering angle for capture into states of principal quantum numbers $n=1$ to 4 . The molecular orientation is at $45^{0}$ with respect to the beam axis. (Note that information on the scattering angle may be obtained either indirectly from the impact parameter dependence of the transition amplitude or directly from the wave picture, see Sec. III C.) Interference patterns in the form of sharp dips can be seen in the scattering angle. They are due to the geometrical structure factor describing scattering phases from identical centers. More complicated structures are expected for subclusters of more than two centers, where our method may be used for qualitative analysis.

\section{E. Large systems}

To complete the theoretical formulation, in this subsection we shall develop the independent subcluster approximation where the wavefunction of the electrons in each of the different subclusters evolves independently from the others.

The total Hamiltonian for the static molecule consists of the sum of the individual subclusters $H_{0, I}(4)$ and their interactions between each other $W_{I, K}$ [see Eq. (A1) in the Appendix] as given by,

$$
H_{0}=\sum_{I=1}^{N} H_{0, I}+\sum_{I=1}^{N} \sum_{\substack{K=1 \\(K \neq I)}}^{N} W_{I, K}
$$

Details of the derivation closely mirror those in subsections $\mathrm{B}$ and $\mathrm{C}$ and can be found in the Appendix. The main 
result is that the total transition amplitude, $A$, for the molecule is again reduced to a product of transition amplitudes, $A_{I}$, for each subcluster when $W_{I, K}$ is averaged or neglected

$$
A \equiv \prod_{I=1}^{N} A_{I} .
$$

The above result is stronger (i.e. has fewer approximations) than the results presented in subsections B and C. If at this point the $A_{I}$ can be evaluated, then Eq. (41) can be used to find the final transition probabilities and cross sections for the molecule or cluster, and the further reduction to individual centers (as illustrated in Fig. 1) is not needed.

If, on the other hand, the independent center, independent electron approximations are successively carried through, the total transition amplitude for the molecule may be similarly obtained from Eqs. (29), (39), and (41)

$$
A \cong \prod_{I=1}^{N} \frac{1}{N_{I} !} \sum_{P} P \prod_{k=1}^{N_{I}} \exp \left\{-i Q_{\min }^{k} R_{I z}^{k}\right\} \prod_{l=1}^{n_{I}} a_{I k}^{l}\left(\mathbf{b}_{I}^{k}\right) .
$$

Equations (33), (39), and (42) are the central results of this paper.

\section{DISCUSSION}

\section{A. Many body effects}

The many body problem is difficult due to the coupling between the constituent particles in a system of particles. It is this coupling (or correlation) which we have eliminated by averaging or simply neglecting it. In our independent center independent electron approximation a molecule or cluster is treated as a collection of independent centers composed of independent electrons. However, molecules are often more than a collection of independent atoms and atoms more than a collection of independent electrons. The advantage of an approximation such as ours is that it is sufficiently simple that it may be applied to collisions in which many constituent particles undergo transitions (e.g. multiple excitation, ionization and transfer of electrons, constituent centers, etc.). Moreover, agreement of our results with experimental observations can often be significantly improved by sensible application of a mean field approximation to various correlation interactions and combination with other compatible methods such as use of simple shake effects. ${ }^{2}$ A model similar to ours, but confined to single electron transitions, is discussed in the well known text of Landau and Liftshitz. ${ }^{4}$ Nonetheless, we wish to emphasize that such simple models are not in general expected to be accurate for molecules or clusters in which interactions between the various centers are significant. In particular chemical bonding and electron correlation, which are often important, are neglected or at best approximated in our development. Thus, we expect application of this simple model to give only qualitative results in cases where interactions between the centers are not relatively weak.

Our independent subcluster approximation is expected to be valid when interactions between the subclusters are small. In particular, if the subclusters are well separated so that bonding energy between subclusters is smaller than the binding energies of the electrons to each center, then this independent subcluster approximation may yield qualitatively accurate results. For example, this independent subcluster approximation may be applied to tightly bound electrons in each subcluster of a diffuse molecule or cluster, where the distance $\mathbf{r}^{i}$ of the electron from the atomic nucleus is small compared to the distance $\left|\mathbf{R}_{I}-\mathbf{R}_{K}\right|$ between subclusters. If the bonds can be well approximated by an overlap of single electronic wave functions from each subcluster, then these effects may be represented by mean field potentials to obtain suitable electronic wavefunctions.

Different subclusters (e.g. the $C_{1}$ and $C_{2}$ subclusters in Fig. 1) are regarded as distinguishable atomic centers. This means that, for example, in $\mathrm{H}_{2} \mathrm{O}$ the $2 \mathrm{H}$ and the $\mathrm{O}$ are considered separately. An electron is either associated with $\mathrm{H}_{2}$ or O. In our approximation such an electron is not shared between different subclusters. However, if two or more centers are the same (e.g. two H's in an $\mathrm{H}_{2}$ subcluster), then one does not distinguish with which atomic center an electron is associated. In the case where two or more identical centers are in different subclusters (e.g. $\mathrm{CH}_{3}-\mathrm{OH}$ has an $\mathrm{H}$ in both $\mathrm{CH}_{3}$ and $\mathrm{OH}$ ) we have neglected the symmetry terms in the identical centers (e.g. H) that are within different subclusters. This is sensible if the subclusters are not too tightly packed. We point out, however, that there are many molecules that do not fit our description easily. DNA, for example, has many identical atoms which are not sensibly grouped together due to their geometry. And in $\mathrm{C}_{8} \mathrm{H}_{18}$ it might not be sensible to consider $\mathrm{C}_{8}$ and $\mathrm{H}_{18}$ as subclusters of identical atoms because of their geometry. On the other hand it is often obvious how to apply the approximations we use on a case by case basis.

Let us consider a specific example of unexpected success of the independent subcluster approximation. If a molecule or cluster has a subunit, e.g. $\left(C_{1}^{2} C_{2}^{2}\right)^{3}$, which has strong bonds and which occurs repeatedly, then the wave function for this subunit is not expected to be well represented by a product of single center wave functions. In addition, significant interference between the repeated subunits is likely if the subunits are not well separated. Based on this example one would not expect the independent center approximation to work particularly well for collisions of bare ions with the molecule $\mathrm{H}_{2}$ or collisions of $\mathrm{H}_{2}^{+}$with atoms. Nevertheless, there is some evidence ${ }^{11}$ that total cross sections for collisions of ions with $\mathrm{H}_{2}$ can be determined to within a factor of two with this simple model (with modified binding energies), and the interference patterns for a two electron transition, namely transfer-excitation, may also be determined within a factor of two. Proposed experimental studies ${ }^{14,15}$ of collisions of $\mathrm{H}_{2}^{+}$ions with atoms will further test the usefulness of this simple approximation.

The independent electron approximation within each center is valid if the electron-electron correlation is relatively weak within each center. This approximation generally is not well justified for the outer atomic shells with many strongly correlated electrons, although use of mean field potential or a screened nuclear charge may yield much better 
results than calculations or estimates done without any consideration of the electron-electron interactions. The independent electron approximation does give good agreement for single and multiple electron transitions of inner-shell electrons with the condition that the binding energy be increased with the degree of ionization if the electrons are not quickly removed. We also note that the independent electron approximation may in some cases apply to electrons localized between individual atoms. The effects of electron exchange terms due to the Pauli principle often seem to be small especially for high velocity collisions. The reason for this is not well understood. Predictions of specific cases that exhibit strong exchange effects have been made, ${ }^{23}$ but these systems have not yet been observed experimentally. More detailed discussions of the independent electron approximation in atomic collisions is given elsewhere..$^{2,18,23}$

\section{B. The frozen approximation}

Another approximation that we have used is to neglect the internal motion of the centers. That is, vibrations and rotations of the molecule are neglected following Eq. (21). If the time of the collision is shorter than the time of rotation and vibration, then this approximation may be justified, and the centers may be regarded as frozen during the collision. This is a key assumption in the impulse approximation for many particle systems discussed in detail by Goldberger and Watson. $^{25}$ While inclusion of vibration and rotation may be possible, in this paper we assume that the collision velocity is large compared to the velocities of the atomic centers and the electrons within the molecule or cluster. In some cases it may be appropriate to average over the rotational (and vibrational) motion of the cluster or molecule.

\section{The geometrical structure factor}

The physical nature of the phase terms in Eq. (31) may be more easily understood by transforming from the impact parameter representation to the wave picture. The probability amplitude, $A(\mathbf{b})$, is generally related to the transition matrix, $T(\mathbf{Q})$, by the relationship, $A(\mathbf{b})=\frac{1}{v} \int e^{i \mathbf{Q}_{\perp} \cdot \mathbf{b}} T(\mathbf{Q}) d \mathbf{Q}_{\perp}$, where $\mathbf{Q}_{\perp}$ is the component of the momentum transfer, $\mathbf{Q}$, perpendicular to the asymptotic velocity of the incoming particle. Following this transformation, the amplitude $A_{I}$ of Eq. (33) is given by

$$
T_{I}=T_{I}(0)\left(\sum_{k}^{N_{I}} e^{-i \mathbf{Q} \cdot \mathbf{R}_{I}^{k}}\right),
$$

where $T_{I}(0)$ is the transition amplitude for a single center, $C_{I}$, located at $\mathbf{R}_{0}=0$. The cross section, $d \sigma$, differential in $\mathbf{Q}$, is proportional to the square of $T$, so that

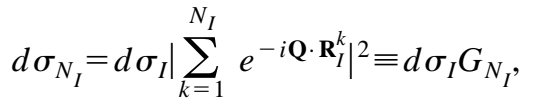

where $G_{N_{I}}$ is a geometrical structure factor containing interference between the $N_{I}$ identical centers and $d \sigma_{I}$ is the differential cross section for scattering from a single center, $C_{I}$. Here, $G_{N_{I}}=N_{I}+\Sigma_{k=1}^{N_{I}} \sum_{j>k} \cos \left(\delta_{I}^{\prime j}-\delta_{I}^{\prime k}\right)$, where
$\delta_{I}^{\prime k}=\mathbf{Q} \cdot \mathbf{R}_{I}^{k}$. At this point Eq. (44) is equivalent to Eq. (19) using Eqs. (31)-(33) because the integral of $\left|T\left(\mathbf{Q}_{\perp}\right)\right|^{2}$ over $\mathbf{Q}_{\perp}$ gives the same total cross section as the integral of $|A(\mathbf{b})|^{2}$ over $\mathbf{b}$. If the transverse momentum transfer, $\mathbf{Q}_{\perp}$, is neglected (e.g. $Q_{\perp} \ll Q_{z}$ ), then $\delta_{I}^{\prime k}=\delta_{I}^{k}$ given by Eq. (32) and the approximations that follow. The factor $G_{N_{I}}$ is the same as the well known geometrical structure factor ${ }^{26,27}$ obtained for classical scattering of waves from $N_{I}$ identical centers. Such a structure factor has also been used to analyze neutron scattering from heavy nuclei, ${ }^{28}$ where the cases of ideal lattices, crystals with thermal disorder and liquids are considered. It is easily shown that as $\mathbf{R}_{I}^{k} \rightarrow 0, G_{N_{I}} \rightarrow N_{I}^{2}$; and as $\mathbf{R}_{I}^{k} \rightarrow \infty$, $G_{N_{I}} \rightarrow N_{I}$. The first limit is fully coherent and the latter is fully incoherent.

There are a few additional points concerning these phases we wish to note. First, addition of phases is consistent with the addition of energies. In the case of independent multiple transitions on a single center the probability amplitude is a product of independent amplitudes [e.g. Eq. (38)] so that the total phase is the sum of the individual phases. Each phase is linear in the transition energy as discussed after Eq. (32). The total transition energy is the sum of the individual transition energies. So the total phase for multiple transitions must be the sum of the individual transition phases in the independent particle approximation. Second, if the mass, $M_{I}^{k}$, of each of the centers is the same, then $\Sigma_{k} \delta_{I}^{k}=Q_{z} \Sigma_{k}\left(R_{I z}^{k}-R_{0 z}\right)=0$ since $\sum_{k} M_{I}^{k}\left(\mathbf{R}_{I}^{k}-\mathbf{R}_{0}\right)=0$ because $\mathbf{R}_{0}$ is the center of mass. If some of the masses differ (e.g. due to different isotopes), then $\Sigma_{k} \delta_{I}^{k}$ sums to a overall non-zero constant phase. Third, our phases occur because our symmetrization gives a superposition of single electron atomic wave functions. Any superposition may lead to phase contributions. Finally we note that in most cases where multiple transitions occur it is easier to approximately evaluate $A$ (B) than $T(\mathbf{Q})$ using our method because $T(\mathbf{Q})$ is not a simple product of simpler terms.

The effect of the interferences due to the identical nature of the centers are observable as illustrated in Fig. 3. ${ }^{12,11,14}$ Such observations may be useful in testing the limits of applicability of our approximations.

\section{Long range interactions}

The interaction that causes the transitions of the target electrons, $V^{\prime}$, was taken in Eqs. (10) and (11) to be the Coulomb interaction between the projectile and the target electrons, namely $-Z_{P} /\left|\mathbf{R}(t)-\mathbf{R}_{I}^{k}-\mathbf{r}_{I}^{k, i}\right|$. All of the interactions of the projectile with each of the nuclei are, in principle, included in the phase terms of the full wave functions in Eqs. (6) and (7). An alternative ${ }^{17}$ that is equivalent in an exact calculation is to use $-Z_{P} /\left|\mathbf{R}(t)-\mathbf{R}_{I}^{k}-\mathbf{r}_{I}^{k, i}\right|+Z_{P} /$ $\left|\mathbf{R}(t)-\mathbf{R}_{I}^{k}\right|$, which pairs off the projectile-electron interaction with a projectile-proton interaction in the target nucleus. That is, the interaction that produces the electronic transitions may be taken to be a short range interaction. This eliminates the Coulomb tail which can lead to mathematical and numerical difficulties. If there are more protons than electrons in the target (i.e. $Z_{I}>n_{I}$ ), then the extra Coulomb 
terms may be included in the determination of the trajectory $\mathbf{R}(t)$. In this latter approach if the target is neutral there are no Coulomb phases at large distances and the trajectory, $\mathbf{R}(\mathrm{t})$, is determined by the short ranged static potential of the total target charge density. In general the target electron motion decouples from the projectile motion to order $\left(m_{e} v_{\text {orbit }} / M_{P} v\right) .^{17}$

\section{E. Simple binomial distributions}

It is often the case that there are transitions with identical (or nearly so) probabilities. This may occur because the probabilities are nearly the same for physical reasons (e.g. electrons in the same atomic shell). Or one may wish to neglect the symmetrization of the quantum wave function and treat the system as a collection of $N$ identical classical particles. Then a binomial coefficient may be used ${ }^{2,6,7}$ to count the number of equivalent ways in which a transition occurs. That is, e.g. there is a factor of $\left(\begin{array}{l}N \\ n\end{array}\right)$ giving the number of different ways in which $\mathrm{n}$ transitions occur in $N$ equivalent centers. Also, simple sum rules may be used to remove from active consideration electrons one may wish to bypass. 2,24

\section{F. Further simplifications}

Two practical points may be worth noting. First, Ben-Itzhak $^{29}$ has shown that under certain conditions first order perturbation theory may be correctly used to evaluate the single electron amplitudes, $a_{I k}^{l}$, even when the system interacts strongly with a highly charged projectile. This means that the evaluation of many electron transitions may be simplified. Second, as a relatively crude approximation, simple shake terms, which lead to some additional final state transitions, may be introduced by using different nonorthogonal basis sets for the asymptotic initial and final state single electron static wave functions. ${ }^{2}$ This may be useful for cases where final state rearrangement of the electrons causes some of the multiple transitions in a reaction.

\section{G. Non-localized projectiles}

In this paper we emphasize the interaction of a molecule or cluster with a heavy charged point particle. In many cases of physical and chemical interest, the projectile may carry electrons. ${ }^{2,30-32}$ In these cases rigorous application of our methods is more difficult because the classical trajectory of the incoming projectile, introduced above Eq. (11), is not well defined since the wavelength of the projectile electrons may be comparable to the size of the interaction region. Also application of the independent electron approximation is difficult, especially for neutral, or nearly neutral projectiles, because often it is not sensible to neglect the interaction between the electrons on the target with the electrons on the projectile since this interaction leads to screening of the nuclear charge, $Z_{p}$, of the projectile by the $n_{p}$ projectile electrons which may be significant. ${ }^{2,31}$ This problem has been solved ${ }^{2,31,32}$ in first order perturbation theory in the wave picture. In this first order approximation one may sim- ply replace the square of the projectile nuclear charge, $Z_{p}^{2}$, by the square of an effective charge, $Z_{p}^{\text {eff } 2}(\mathbf{Q})$, which depends on the momentum transfer, $\mathbf{Q}$, of the projectile. For small $\mathbf{Q}$ which corresponds to collisions at distances large compared to the distance of the projectile electrons from their nucleus, $Z_{p}^{\text {eff } 2} \rightarrow\left(Z_{p}-n_{P}\right)^{2}$, so that the projectile acts as a point particle of charge $\left(Z_{p}-n_{p}\right)$. For large $\mathbf{Q}$ where the projectile electrons are well separated from the projectile nucleus, $Z_{p}^{\text {eff } 2} \rightarrow Z_{p}^{2}+n_{p}$, which corresponds to independent scattering by the various charged particles on the projectile. Both limits are physically sensible. In particular, for one electron projectile ions in their ground state, $Z_{p}^{\text {eff } 2}=Z_{p}^{2}+1-2 Z_{p} /\left(1+Q^{2} / 2 Z_{p}\right)^{2}$. Extension to many electron projectiles has also been considered. ${ }^{2}$ While this result is rigorously valid only within the limitations of first order perturbation theory in the wave picture, a relatively simple model containing the same physically sensible limits is easily obtained by making a classical transformation from the momentum transfer, $\mathbf{Q}$, to the impact parameter, $\mathbf{b}$, and using $Z_{p}^{\text {eff }}(\mathbf{b})$ in place of the corresponding bare charge, $Z_{p}$, in the probability amplitude of Eq. (19) and the approximations that follow. It should be noted, however, that the correct scattering probability may not exceed unity, and that a rigorous first order amplitude ${ }^{33-35}$ is given by a convolution over a virtual impact parameter, $\mathbf{b}^{\prime}$, of the probability amplitude for a point charge, $A\left(\mathbf{b}^{\prime}\right)$, over $Z_{P}^{\text {eff }}\left(\mathbf{b}^{\prime}-\mathbf{b}\right)$.

Extension of our methods to projectiles that themselves have multiple centers may also be possible. In limiting cases where the projectile centers are well separated or very compact, it is valid to neglect interactions between centers on the target and interactions between centers on the projectile and to include interactions between centers of the target and the projectile. Symmetry between like centers on the projectile and target may also be included.

\section{SUMMARY}

We have considered a molecule or cluster with $N_{C}=\sum_{I}^{N} N_{I}$ centers, $C_{I}$ [Sec. II A]. This molecule or cluster is denoted by $C_{1}^{N_{1}} C_{2}^{N_{2}} \ldots C_{I}^{N_{I}} \ldots C_{N}^{N_{N}}$, where $C_{I}^{N_{I}}$ denotes a subcluster of $N_{I}$ identical centers, $C_{I}$. The key approximations are as follows.

(1) The independent subcluster approximation [Sec. II E]: The interaction between different subclusters is neglected so that $A \approx \prod_{I}^{N} A_{I}$.

(2) Coherent scattering from identical centers within subclusters [Sec. II B]: Scattering of the wave fronts of the projectile from multiple identical independent centers is included within each subcluster so that $A_{I}=\left(1 / N_{I} !\right) \Sigma_{P} P \prod_{k=1}^{N_{I}} e^{i \delta_{I}^{k}} A_{I k}\left(\mathbf{b}_{I}^{k}\right)$, where $\Sigma_{P} P$ is the permutation operator. The $\delta_{I}^{k}$ phase terms are related to the usual geometrical structure factor.

(3) The independent electron approximation [Sec. II C]: The correlation between electrons within each center is neglected so that $A_{I k} \approx \prod_{i=1}^{n_{I}} a_{I k}^{i}$, and $\delta_{I}^{k} \approx \sum_{i}^{n_{I}} \delta_{I}^{k i}$.

Our independent center independent electron approximation (ICEA), in the absence of the coherence terms in step 2 
above, corresponds to a simple generalization of the static Hartree product wave function to a dynamic probability amplitude using a single basis set, $\{\phi\}$. The more complete time-dependent Hartree-Fock (TDHF) approximation is a dynamic generalization of the static Hartree-Fock approximation that fully includes the effects of electron symmetry. TDHF is evaluated variationally for each time during the scattering event, so that $\{\phi\}$ changes continuously with time. While TDHF is a less approximate uncorrelated limit of multi-particle scattering than our ICEA, TDHF is more difficult to implement than our simpler ICEA.

\section{CONCLUSION}

A method has been developed for evaluating probabilities and cross sections for multiple-electron transitions in the interaction of molecules or clusters with various charged partners. The probability amplitude, as well as the transition probability, is expressed as a product of independent center probabilities including phase terms for identical centers. Each of the probability amplitudes for the atomic centers may then be expressed as a product of amplitudes for each of the independent electrons. In this independent center independent electron approximation we neglect correlation and some exchange effects which are important in many molecular systems. On the other hand large systems may be described simply.

\section{ACKNOWLEDGMENTS}

We acknowledge useful discussion with I. Ben-Itzhak. This work was supported by the Division of Chemical Sciences, Office of Basic Energy Sciences, Office of Energy Research, U.S. Department of Energy.

\section{APPENDIX: GENERALIZATION TO LARGE SYSTEMS}

This Appendix contains details on independent subcluster approximation presented in Sec. II E. The Hamiltonian for the static molecule as expressed in Eqs. (4) and (40) includes interactions between subclusters, $W_{I, K}$, given by

$$
\begin{aligned}
W_{I, K}= & \frac{1}{2} \sum_{k=1}^{N_{I}} \sum_{j=1}^{N_{K}}\left\{\frac{Z_{I} Z_{K}}{\left|\mathbf{R}_{I}^{k}-\mathbf{R}_{K}^{j}\right|}-\sum_{i=1}^{n_{K}} \frac{Z_{I}}{\left|\mathbf{R}_{I}^{k}-\mathbf{R}_{K}^{j}-\mathbf{r}_{K}^{j, i}\right|}\right. \\
& -\sum_{i=1}^{n_{I}} \frac{Z_{K}}{\left|\mathbf{R}_{I}^{k}+\mathbf{r}_{I}^{k, i}-\mathbf{R}_{K}^{j}\right|} \\
& \left.+\sum_{l=1}^{n_{K}} \frac{1}{\left|\mathbf{R}_{I}^{k}+\mathbf{r}_{I}^{k, i}-\mathbf{R}_{K}^{j}-\mathbf{r}_{K}^{j, l}\right|}\right\}(I \neq K) .
\end{aligned}
$$

The four terms in Eq. (A1) describe, in their respective order, the nuclear-nuclear (first term), nuclear-electron (second and third), and electron-electron (fourth) interactions between subclusters $I$ and $K$. Accordingly, the interaction of the whole molecule with the projectile will also be replaced by the sum of interactions with individual subclusters Eq. (14)

$$
V^{\prime}=\sum_{I=1}^{N} V_{I}
$$

Each subcluster will evolve independently if the interaction between them $W_{I, K}$ is replaced by a mean field approximation, or simply neglected. In the latter case we have

$$
H_{0} \cong \sum_{I=1}^{N} H_{0, I}
$$

With this approximation, we have using Eq. (16) that

$$
\begin{aligned}
V^{\prime}(t)=e^{i H_{0} t} V^{\prime} e^{-i H_{0} t} & =\sum_{I=1}^{N}\left[e^{i H_{0, I^{t}}} V_{I} e^{-i H_{0, I^{t}}}\right] \\
& \equiv \sum_{I=1}^{N} V_{I}(t)
\end{aligned}
$$

where $V_{I}(t)$ now operates on a single subcluster.

As was done in Secs. II B and C Eqs. (23) and (36), one may factor the evolution operator for the molecule as

$$
\begin{aligned}
U\left(t, t_{0}\right) & =T \exp \left[-i \sum_{I=1}^{N} \int_{t_{0}}^{t} V_{I}(t) d t\right] \\
& =\prod_{I=1}^{N} T \exp \left[-i \int_{t_{0}}^{t} V_{I}(t) d t\right] \\
& \equiv \prod_{I=1}^{N} U_{I}\left(t, t_{0}\right) .
\end{aligned}
$$

By neglecting the $W_{I, K}$ terms in the full Hamiltonian that interconnect the subclusters, the evolution operator, $U\left(t, t_{0}\right)$, has become a product of single subcluster evolution operators $U_{I}\left(t, t_{0}\right)$.

Within the present approximation, the initial (final) asymptotic electronic wave function of the cluster, $\phi_{i}\left(\phi_{f}\right)$, is written as a product of single subcluster wave functions, $\phi_{I i}\left(\phi_{I f}\right)$, i.e.

$$
\phi_{i, f}=\prod_{I=1}^{N} \phi_{I i, f} .
$$

Then, from Eqs. (A5) and (A6) using the orthogonality of the $\phi_{I}$ 's and single cluster nature of the $U_{I}$ operators, one has for the final result used in Sec. II E, Eq. (41) that

$$
A=\left\langle\prod_{I=1}^{N} \phi_{I f}\left|\prod_{I=1}^{N} U_{I}\left(t, t_{0}\right)\right| \prod_{I=1}^{N} \phi_{I i}\right\rangle \equiv \prod_{I=1}^{N} A_{I}
$$

and

$$
A_{I}=\left\langle\phi_{I f}\left|U_{I}\left(t, t_{0}\right)\right| \phi_{I i}\right\rangle .
$$

Here $A_{I}$ is the probability amplitude for a particular transition in the subcluster of type I. Eq. (A7) is obtained for ionization and electron capture processes in the case that we can identify in which subcluster the transition occurs. 
${ }^{1}$ N. Stolterfoht, Phys. Scr. 42, 192 (1990); T46, 22 (1993).

${ }^{2}$ J. H. McGuire, Adv. Atom. Mol. Opt. Phys. 29, 217 (1991).

${ }^{3}$ Y. D. Wang, Ph.D. thesis, Tulane University, 1992.

${ }^{4}$ L. D. Landau and E. M. Liftshitz, Quantum Mechanics (Addison-Wesley, Reading, MA, 1958),

${ }^{5}$ M. Gryzinski, Phys. Rev. 138, A349 (1965).

${ }^{6}$ J. M. Hansteen and O. P. Mosebeek, Phys. Rev. Lett. 29, 1961 (1972); J. M. Hansteen, A. M. Johansen, and L. Kocbach, At. Data Nucl. Data Tables 15, 305 (1975).

${ }^{7}$ J. H. McGuire and O. L. Weaver, Phys. Rev. A 14, 41 (1977).

${ }^{8}$ R. N. Zare, J. Chem. Phys. 47, 204 (1967).

${ }^{9}$ T. F. Tuan and E. Gerjuoy, Phys. Rev. 117, 756 (1960).

${ }^{10}$ A. Belkacem, E. P. Kanter, R. E. Mitchell, Z. Vager, and B. J. Zabransky, Phys. Rev. Lett. 63, 2555 (1989).

${ }^{11}$ S. Cheng, C. L. Cocke, V. Frohne, E. Y. Kamber, and S. L. Varghese, Nucl. Instrum. Methods 56/57, 78 (1991); S. Cheng et al. (in preparation).

${ }^{12}$ A. K. Edwards, R. A. Wood, M. A. Magnan and R. A. Ezell, Phys. Rev. A 46, 6970 (1992).

${ }^{13}$ K. Wohrer, G. Sampoll, R. L. Watson, M. Chabot, O. Heber, and V. Horvat, Phys. Rev. A 46, 3929 (1992), and references therein.

${ }^{14}$ I. Ben-Itzhak et al. (private communication).

${ }^{15}$ H. O. Lutz (private communication).

${ }^{16}$ S. E. Corchs, R. D. Rivarola, and J. H. McGuire, Phys. Rev. A 47, 3937 (1993).

${ }^{17}$ J. H. McGuire and O. L. Weaver, Phys. Rev. A 34, 2473 (1986).

${ }^{18}$ J. H. McGuire, Phys. Rev. A 36, 1114 (1987).

${ }^{19}$ A. Messiah, Quantum Mechanics (Wiley, New York, 1965), Chap. XIX, Sec. 24.
${ }^{20}$ Y. D. Wang, J. H. McGuire, and R. D. Rivarola, Phys. Rev. A 40, 3673 (1989).

${ }^{21}$ R. Shingal and C. D. Lin, Phys. Rev. A 40, 1302 (1989).

${ }^{22}$ M. R. C. McDowell and J. P. Coleman, Introduction to the Theory of Ion-Atom Collisions (North-Holland, Amsterdam, 1970), Chaps. 7 and 8.

${ }^{23}$ J. F. Reading and A. L. Ford, Phys. Rev. A 21, 124 (1980).

${ }^{24}$ J. H. McGuire and J. R. Macdonald, Phys. Rev. A 11, 146 (1975).

${ }^{25}$ M. L. Goldberger and K. M. Watson, Collision Theory (Wiley, New York, 1964), Chap. 11

${ }^{26}$ B. Sanger, Z. Phys. D 9, 79 (1988).

${ }^{27}$ Charles Kittel, Introduction to Solid State Physics, 2nd ed. (Wiley, New York, 1956), p. 54.

${ }^{28}$ G. Placzek, B. R. A. Nijbor, and L. Van Hove, Phys. Rev. 82, 392 (1951).

${ }^{29}$ I. Ben-Itzhak, T. J. Gray, J. C. Legg, and J. H. McGuire, Phys. Rev. A 37, 3685 (1988)

${ }^{30}$ T. J. M. Zouros, D. H. Lee, J. M. Sanders, and P. Richard, Nucl. Instru. Meth. B 79, 166 (1993).

${ }^{31}$ E. C. Montenegro, S. Melo Wilson, W. E. Meyerhof, and A. G. de Pinho, Phys. Rev. Lett. 69, 3033 (1992).

${ }^{32}$ E. C. Montenegro, W. E. Meyerhof, and J. H. McGuire, Adv. Atom. Mol. Opt. Phys. 34, 249 (1994)

${ }^{33} \mathrm{~J}$. H. McGuire and E. C. Montenegro, Abstracts of Contributed Papers of the XVIII International Conference on the Physics of Electronic and Atomic Collisions, edited by T. Andersen, B. Fastrup, F. Folkmann, and H. Knudsen (Aarhus, Denmark, 1993), p. 656.

${ }^{34}$ S. Ricz, B. Sulik, and N. Stolterfoht, Phys. Rev. A 47, 1930 (1993).

${ }^{35}$ J. Wang, J. H. McGuire, and E. C. Montenegro, Phys. Rev. A 51, 504 (1995) 